\title{
Non-symmetric approximations of functional classes by splines on the real line
}

\section{Parfinovych N.V.}

Let $S_{h, m}, h>0, m \in \mathbb{N}$, be the spaces of polynomial splines of order $m$ of deficiency 1 with nodes at the points $k h, k \in \mathbb{Z}$.

We obtain exact values of the best $(\alpha, \beta)$-approximations by spaces $S_{h, m} \cap L_{1}(\mathbb{R})$ in the space $L_{1}(\mathbb{R})$ for the classes $W_{1,1}^{r}(\mathbb{R}), r \in \mathbb{N}$, of functions, defined on the whole real line, integrable on $\mathbb{R}$ and such that their $r$ th derivatives belong to the unit ball of $L_{1}(\mathbb{R})$.

These results generalize the well-known G.G. Magaril-Ilyaev's and V.M. Tikhomirov's results on the exact values of the best approximations of classes $W_{1,1}^{r}(\mathbb{R})$ by splines from $S_{h, m} \cap L_{1}(\mathbb{R})$ (case $\alpha=\beta=1$ ), as well as are non-periodic analogs of the V.F. Babenko's result on the best nonsymmetric approximations of classes $W_{1}^{r}(\mathbb{T})$ of $2 \pi$-periodic functions with $r$ th derivative belonging to the unit ball of $L_{1}(\mathbb{T})$ by periodic polynomial splines of minimal deficiency.

As a corollary of the main result, we obtain exact values of the best one-sided approximations of classes $W_{1}^{r}$ by polynomial splines from $S_{h, m}(\mathbb{T})$. This result is a periodic analogue of the results of A.A. Ligun and V.G. Doronin on the best one-sided approximations of classes $W_{1}^{r}$ by spaces $S_{h, m}(\mathbb{T})$.

Key words and phrases: best $L_{1}$-approximation, one-sided approximation, non-symmetric approximation, polynomial spline, functional class.

Oles Honchar Dnipro National University, 72 Gagarin avenue, 49010, Dnipro, Ukraine

E-mail: nat-vic-parei.ua

\section{Introduction}

Let $\mathbb{N}, \mathbb{Z}_{+}, \mathbb{Z}, \mathbb{R}_{+}$, and $\mathbb{R}$ be the sets of integer positive, integer non-negative, integer, real non-negative, and real numbers, respectively, and $\mathbb{T}$ be the interval $[0,2 \pi]$ with identified ends.

Let $L_{p}(\mathbb{G}), 1 \leq p \leq \infty, \mathbb{G} \subset \mathbb{R}$, be the spaces of all measurable on $\mathbb{G}$ functions with norms $\|\cdot\|_{L_{p}(\mathbb{G})}, C^{r}(\mathbb{G}), r \in \mathbb{Z}_{+}$, be the spaces of $r$ times continuously differentiable (continuous for $r=0$ ) on $\mathbb{G}$ functions, and $A C(\mathbb{G})$ be the set of all absolutely continuous (locally for $\mathbb{G}=\mathbb{R}$ and $\mathbb{G}=\mathbb{R}_{+}$) on $\mathbb{G}$ functions.

For $f \in L_{p}(\mathbb{G})$ and $\alpha, \beta>0$ we set

$$
\|f\|_{L_{p}(\mathrm{G}) ; \alpha, \beta}=\left\|\alpha f_{+}+\beta f_{-}\right\|_{L_{p}(\mathrm{G})},
$$

where $f_{ \pm}(t)=\max \{ \pm f(t), 0\}$. The quantity

$$
E(f, H)_{L_{p}(\mathbb{G}) ; \alpha, \beta}:=\inf _{u \in H}\|f-u\|_{L_{p}(\mathbb{G}) ; \alpha, \beta}
$$

is called the best $(\alpha, \beta)$-approximation of a function $f \in L_{p}(\mathbb{G})$ by the set $H \subset L_{p}(\mathbb{R})$ in the metric $L_{p}(\mathbb{G})$. 
The notation $E(f)_{L_{p}(G) ; \alpha, \beta}$ will be used for the best $(\alpha, \beta)$-approximation of a function $f \in L_{p}(\mathbb{G})$ by subset of constants.

For class of functions $M \subset L_{p}(\mathbb{G})$, the quantity

$$
E(M, H)_{L_{p}(\mathbb{G}) ; \alpha, \beta}:=\sup _{f \in M} E(f, H)_{L_{p}(\mathbb{G}) ; \alpha, \beta}
$$

is called the best $(\alpha, \beta)$-approximation of the class $M$ by the set $H$ in the space $L_{p}(G)$. If $\alpha=\beta=1$ the quantities (1) and (2) coincide with ordinary best $L_{p}$-approximation of a function $f$ (notation $\left.E(f, H)_{L_{p}(\mathrm{G})}\right)$ and of class $M$ (notation $\left.E(M, H)_{L_{p}(\mathrm{G})}\right)$, respectively.

Let the set $H \subset L_{p}(\mathbb{G})$ be fixed. We associate with the function $f$ the subsets

$$
H_{f}^{+}=\{u(t): u \in H, u(t) \leq f(t), t \in \mathbb{G}\} \quad \text { and } \quad H_{f}^{-}=\{u(t): u \in H, u(t) \geq f(t), t \in \mathbb{G}\} .
$$

For $f \in L_{p}(\mathbb{G})$ and $M \subset L_{p}(\mathbb{G})$ we set

$$
E^{ \pm}(f, H)_{L_{p}(\mathbb{G})}= \begin{cases}\inf \left\{\|f-u\|_{L_{p}(\mathbb{G})}: u \in H_{f}^{ \pm}\right\}, & H_{f}^{ \pm} \neq \varnothing \\ \infty, & H_{f}^{ \pm}=\varnothing\end{cases}
$$

and

$$
E^{ \pm}(M, H)_{L_{p}(\mathbb{G})}=\sup _{f \in M} E^{ \pm}(f, H)_{L_{p}(\mathbb{G})}
$$

Quantities $E^{ \pm}(f, H)_{L_{p}(\mathbb{G})}$ and $E^{ \pm}(M, H)_{L_{p}(\mathbb{G})}$ are called the best approximation from below $(+)$ and from above $(-)$ of a function $f \in L_{p}(\mathbb{G})$ and $M \subset L_{p}(\mathbb{G})$, respectively.

In the case when $\mathbb{G}$ is a segment, V.F. Babenko [1] (see also [7, Theorems 1.4.10 and 1.5.9]) established that if the set $H \subset L_{p}(\mathbb{G}), 1 \leq p \leq \infty$, is locally compact then for any function $f \in L_{p}(\mathbb{G})$, monotonously on $\alpha$ and $\beta$

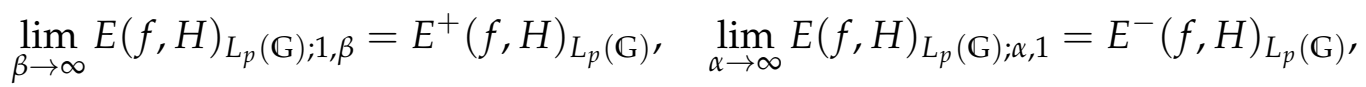

for any set $M \subset L_{p}(\mathbb{G})$, monotonously on $\alpha$ and $\beta$

$$
\lim _{\beta \rightarrow \infty} E(M, H)_{L_{p}(\mathbb{G}) ; 1, \beta}=E^{+}(M, H)_{L_{p}(\mathbb{G})}, \quad \lim _{\alpha \rightarrow \infty} E(M, H)_{L_{p}(\mathrm{G}) ; \alpha, 1}=E^{-}(M, H)_{L_{p}(\mathbb{G})} .
$$

In the case $\mathbb{G}=\mathbb{R}$ one can prove (3) and (4) the same.

For $r \in \mathbb{N}$, let

$$
\begin{gathered}
L_{p}^{r}(\mathbb{G})=\left\{f \in L_{1}(\mathbb{G}): f^{(r-1)} \in A C(\mathbb{G}), f^{(r)} \in L_{p}(\mathbb{G})\right\}, \\
W_{p}^{r}(\mathbb{G})=\left\{f \in L_{p}^{r}(\mathbb{G}):\left\|f^{(r)}\right\|_{L_{p}(\mathbb{G})} \leq 1\right\}, \quad W_{p, q}^{r}(\mathbb{G})=W_{p}^{r}(\mathbb{G}) \cap L_{q}(\mathbb{G}) .
\end{gathered}
$$

For $h>0$ and $m \in \mathbb{Z}_{+}$, by $S_{h, m}(\mathbb{R})$ we denote the collection of functions $s \in C^{(m-1)}(\mathbb{R}), m \geq 1$, such that $\left.s^{(m)}\right|_{(j h ;(j+1) h)}=c_{j}=$ const, $j \in \mathbb{Z}$. The set $S_{h, m}(\mathbb{R})$ is called the space of polynomial splines on $\mathbb{R}$ of order $m$ and defect 1 with nodes at the points $j h, j \in \mathbb{Z}$.

The subspace of $2 \pi$-periodic polynomial splines of order $m$ and defect 1 with nodes at the points $j \pi / n, j \in \mathbb{Z}$, we denote as $S_{\pi / n, m}(\mathbb{T})$. 
For $\alpha, \beta>0, \lambda>0, m \in \mathbb{N}$, by $\varphi_{\lambda, m}(\alpha, \beta ; t)$ we denote $(2 \pi / \lambda)$-periodic integral of order $m$ with zero mean over the period from even $(2 \pi / \lambda)$-periodic function $\varphi_{\lambda, 0}(\alpha, \beta ; t)$, which for $t \in[0, \pi / n)$ is defined as follows

$$
\varphi_{\lambda, 0}(\alpha, \beta ; t)= \begin{cases}\alpha, & 0 \leq t \leq \pi \beta /(\lambda(\alpha+\beta)), \\ -\beta, & \pi \beta /(\lambda(\alpha+\beta))<t<\pi / n .\end{cases}
$$

For $\alpha=\beta=1$ instead of $\varphi_{\lambda, m}(\alpha, \beta ; t)$ we will write $\varphi_{\lambda, m}(t)$.

By

$$
B_{\lambda, m}(t)=-2 \lambda^{-m} \sum_{k=1}^{n} \frac{\cos (k \lambda t-\pi m / 2)}{k^{m}}, \quad m \in \mathbb{N},
$$

we denote the Bernoulli kernel of order $m$ (see [7, p. 107]).

Note (see [7, p.109]) that for $m \geq 2$ and $\beta \rightarrow \infty$ one has

$$
\left\|\varphi_{1, m}(1, \beta ; \cdot)-B_{\lambda, m}\right\|_{L_{\infty}(\mathbb{T})} \rightarrow 0 \text {. }
$$

Moreover, for all $m \in \mathbb{N}$ and $\beta \rightarrow \infty$,

$$
E\left(\varphi_{1, m}(1, \beta ; \cdot)\right)_{L_{\infty}(\mathbb{T})} \rightarrow E\left(B_{1, m}\right)_{L_{\infty}(\mathbb{T})}
$$

\section{Preliminary information and main results}

Let $p=1, \infty$. It is well known, that for $n, r, m \in \mathbb{N}, m \geq r-1$,

$$
E\left(W_{p}^{r}(\mathbb{T}), S_{\pi / n, m}(\mathbb{T})\right)_{L_{p}(\mathbb{T})}=\frac{\left\|\varphi_{1, r}\right\|_{L_{\infty}(\mathbb{T})}}{n^{r}}
$$

In the case $p=\infty$ and $m=r-1$, the equality (7) was established by V.M. Tikhomirov [14] and in the other cases by A.A. Ligun [8].

Similar results for one-sided approximations of classes $W_{p}^{r}(\mathbb{T})$ by splines were obtained by V.G. Doronin and A.A. Ligun [6]. They proved that for $n, m, r \in \mathbb{N}, m \geq r$,

$$
E^{ \pm}\left(W_{1}^{r}(\mathbb{T}), S_{\pi / n, m}(\mathbb{T})\right)_{L_{1}(\mathbb{T})}=\frac{E\left(B_{1, r}\right)_{L_{\infty}(\mathbb{T})}}{n^{r}} .
$$

Later V.F. Babenko [1] established, that for $n, m, r \in \mathbb{N}, m \geq r$, and $\alpha, \beta>0$,

$$
E^{ \pm}\left(W_{1}^{r}(\mathbb{T}), S_{\pi / n, m}(\mathbb{T})\right)_{L_{1}(\mathbb{T}) ; \alpha, \beta}=\frac{E\left(\varphi_{1, r}(\alpha, \beta ; \cdot)\right)_{L_{\infty}(\mathbb{T})}}{n^{r}} .
$$

Note that considering (5), (6), the result (8) can be obtained from (9) by passing to the limit.

For other results on best approximations of classes $W_{1}^{r}(\mathbb{T})$ by splines in a periodic case see $[2-5,11,12]$ and references therein.

In was proved in [9] that for all $r \in \mathbb{N}, m \in \mathbb{Z}_{+}, m \geq r-1$, and $h>0$,

$$
E\left(W_{1,1}^{r}(\mathbb{R}), S_{h, m}(\mathbb{R}) \cap L_{1}(\mathbb{R})\right)_{L_{1}(\mathbb{R})}=\frac{\left\|\varphi_{1, r}\right\|_{L_{\infty}(\mathbb{T})} \cdot h^{r}}{\pi^{r}} .
$$

Moreover, it is shown in [10] that this result can be obtained from its periodic analogue (7).

In this paper, we obtain non-symmetric analogs of equality (10), based on the result of V.F. Babenko (9).

Theorem. Let $\alpha, \beta>0, r, n, m \in \mathbb{N}, m \geq r, h>0$. Then

$$
E\left(W_{1,1}^{r}(\mathbb{R}), S_{h, m}(\mathbb{R}) \cap L_{1}(\mathbb{R})\right)_{L_{1}(\mathbb{R}) ; \alpha, \beta}=\frac{E\left(\varphi_{1, r}(\alpha, \beta ; \cdot)\right)_{L_{\infty}(\mathbb{T})} \cdot h^{r}}{\pi^{r}} .
$$

Corollary. Let $\alpha, \beta>0, r, n, m \in \mathbb{N}, m \geq r, h>0$. Then

$$
E^{ \pm}\left(W_{1,1}^{r}(\mathbb{R}), S_{h, m}(\mathbb{R}) \cap L_{1}(\mathbb{R})\right)_{L_{1}(\mathbb{R})}=\frac{E\left(B_{1, r}\right)_{L_{\infty}(\mathbb{T})} h^{r}}{\pi^{r}} .
$$




\section{Proof of the main result}

We give a proof of our theorem.

Proof. We obtain an upper bound first. In doing so, we will use ideas and methods from [10]. Let $n \in \mathbb{N}$ and $h>0$. Let us consider the class $W_{p}^{r}\left(\frac{n h}{\pi} \mathbb{T}\right)$ of $2 n h$-periodic functions. Note that $f \in W_{p}^{r}\left(\frac{n h}{\pi} \mathbb{T}\right)$ if and only if $g(t)=f\left(\frac{n h}{\pi} t\right) \in\left(\frac{n h}{\pi}\right)^{r} \cdot W_{p}^{r}(\mathbb{T})$. Similarly, $s \in S_{h, m}\left(\frac{n h}{\pi} \mathbb{T}\right)$ if and only if $\sigma(t)=s\left(\frac{n h}{\pi} t\right) \in S_{h, m}(\mathbb{T})$. Then from (9) we obtain

$$
\begin{aligned}
E\left(W_{p}^{r}\left(\frac{n h}{\pi} \mathbb{T}\right), S_{h, m}\left(\frac{n h}{\pi} \mathbb{T}\right)\right)_{L_{p}\left(\frac{n h}{\pi} \mathbb{T}\right) ; \alpha, \beta} & =\left(\frac{n h}{\pi}\right)^{r} E\left(W_{p}^{r}(\mathbb{T}), S_{h, m}(\mathbb{T})\right)_{L_{p}(\mathbb{T}) ; \alpha, \beta} \\
& =\frac{E\left(\varphi_{1, r}(\alpha, \beta ; \cdot)\right)_{L_{\infty}(\mathbb{T})} \cdot h^{r}}{\pi^{r}} .
\end{aligned}
$$

Let now $f \in W_{1,1}^{r}(\mathbb{R})$ and $\eta(\cdot)$ infinitely differentiable on $\mathbb{R}$ function such that $0 \leq \eta(t) \leq 1$, $t \in \mathbb{R}$, supp $\eta(\cdot) \subset[-1,1]$ and $\eta(t)=1$ subject to $t \in[-1 / 2,1 / 2]$. For any $l>0$ put $\eta_{l}(t):=\eta(t / l)$ and $f_{l}(\cdot)=f(\cdot) \eta_{l}(\cdot)$. By Leibniz's formula we have

$$
f_{l}^{(r)}(t)=\sum_{j=0}^{r}\left(\begin{array}{l}
r \\
j
\end{array}\right) f^{(j)}(t) l^{-(r-j)} \eta_{l}^{(r-j)}(t)
$$

Stain's inequality [13] implies the boundedness of the derivatives $x^{(j)}, j=1,2, \ldots, r-1$, in $L_{1}(\mathbb{R})$. From here and from (12) we obtain

$$
\left\|f_{l}^{(r)}\right\|_{L_{1}(\mathbb{R})} \leq \rho(l)+\left\|f^{(r)}\right\|_{L_{1}(\mathbb{R})} \leq \rho(l)+1,
$$

where $\rho(l) \rightarrow 0$ as $l \rightarrow \infty$.

By $\tilde{f}_{h}$ denote $4 n h$-periodic continuation of function $(\rho(2 n h)+1)^{-1} f_{2 n h}(\cdot) \subset W_{[-2 n h, 2 n h]}^{r}$ and $\left\|\tilde{f}_{h}^{(r)}\right\|_{L_{p}\left(\frac{n h}{\pi} \mathbb{T}\right)} \leq 1$. Thus $\tilde{f}_{h} \in W_{p}^{r}\left(\frac{n h}{\pi} \mathbb{T}\right)$. Since $S_{h, m}\left(\frac{n h}{\pi} \mathbb{T}\right)$ is a finite-dimensional space, there exists a spline $s_{n}^{*}$ from this space such that

$$
\left\|\tilde{f}_{h}-s_{n}^{*}\right\|_{L_{p}\left(\frac{n h}{\pi} \mathbb{T}\right) ; \alpha, \beta}=E\left(\tilde{f}_{h}, S_{h, m}\left(\frac{n h}{\pi} \mathbb{T}\right)\right)_{L_{p}\left(\frac{n h}{\pi} \mathbb{T}\right) ; \alpha, \beta}
$$

Since

$$
E\left(\tilde{f}_{h}, S_{h, m}\left(\frac{n h}{\pi} \mathbb{T}\right)\right)_{L_{1}\left(\frac{n h}{\pi} \mathbb{T}\right) ; \alpha, \beta} \leq\left\|\tilde{f}_{h}\right\|_{L_{1}\left(\frac{n h}{\pi} \mathbb{T}\right) ; \alpha, \beta^{\prime}}
$$

using the inequality

$$
\min \{\alpha, \beta\}\|\cdot\|_{L_{p}(\mathbb{G})} \leq\|\cdot\|_{L_{p}(\mathbb{G}) ; \alpha, \beta} \leq \max \{\alpha, \beta\}\|\cdot\|_{L_{p}(\mathbb{G})}
$$

we get

$$
\min \{\alpha, \beta\}\left\|s_{n}^{*}\right\|_{L_{1}\left(\frac{n h}{\pi} \mathbb{T}\right)} \leq\left\|s_{n}^{*}-\tilde{f}_{h}+\tilde{f}_{h}\right\|_{L_{1}\left(\frac{n h}{\pi} \mathbb{T}\right) ; \alpha, \beta} \leq 2\left\|\tilde{f}_{h}\right\|_{L_{1}\left(\frac{n h}{\pi} \mathbb{T}\right) ; \alpha, \beta} \leq 2 \max \{\alpha, \beta\}\left\|\tilde{f}_{h}\right\|_{L_{1}\left(\frac{n h}{\pi} \mathbb{T}\right)^{\prime}}
$$

whence

$$
\left\|s_{n}^{*}\right\|_{L_{1}\left(\frac{n h}{\pi} \mathbb{T}\right)} \leq \frac{2 \max \{\alpha, \beta\}}{\min \{\alpha, \beta\}}\left\|\tilde{f}_{h}\right\|_{L_{1}\left(\frac{n h}{\pi} \mathbb{T}\right)} .
$$

Thus the conditions of Proposition 1 in [10] are satisfied. According to them there exists a spline $\xi \in S_{h, m}(\mathbb{R}) \cap L_{1}(\mathbb{R})$ and a sequence $\left\{s_{n_{j}}^{*}\right\}_{j \in \mathbb{N}}$ such that $\left\|\xi-s_{n_{j}}^{*}\right\|_{L_{\infty}(I)} \rightarrow 0, j \rightarrow \infty$, for any finite segment $I \subset \mathbb{R}$. 
We set $a_{n}=\rho(2 n h)+1$. Taking into account that $\left.\tilde{f}_{h}(\cdot)\right|_{[-n h, n h]}=a_{n}^{-1} f(\cdot)$ for all $n \in \mathbb{N}$ and for all $j \in \mathbb{N}$ such that $n_{j} \geq n$, based on (11) we will have

$$
\begin{aligned}
\left\|f-s_{n_{j}}^{*}\right\|_{L_{1}([-n h, n h]) ; \alpha, \beta} & \leq\left\|a_{n_{j}} \tilde{f}_{n_{j}}-s_{n_{j}}^{*}\right\|_{L_{1}\left(\left[-n_{j} h, n_{j} h\right]\right) ; \alpha, \beta} \\
& \leq a_{n_{j}} E\left(W_{1}^{r}\left(\frac{n h}{\pi} \mathbb{T}\right), S_{h, m}\left(\frac{n h}{\pi} \mathbb{T}\right)\right)_{L_{1}\left(\frac{n h}{\pi} \mathbb{T}\right) ; \alpha, \beta} \\
& =a_{n_{j}} \frac{E\left(\varphi_{1, r}(\alpha, \beta ; \cdot)\right)_{L_{\infty}(\mathbb{R})} h^{r}}{\pi^{r}}
\end{aligned}
$$

Passing in (13) to the limit as $j \rightarrow \infty$, according to [10, Proposition 1] we obtain

$$
\|\tilde{f}-\xi\|_{L_{1}([-n h, n h]) ; \alpha, \beta} \leq \frac{E\left(\varphi_{1, r}(\alpha, \beta ; \cdot)\right)_{L_{\infty}(\mathbb{R})} h^{r}}{\pi^{r}} .
$$

Whence, taking into account Fatou's lemma, we have

$$
\|\tilde{f}-\xi\|_{L_{1}(\mathbb{R}) ; \alpha, \beta} \leq \frac{E\left(\varphi_{1, r}(\alpha, \beta ; \cdot)\right)_{L_{\infty}(\mathbb{R})} h^{r}}{\pi^{r}} .
$$

The upper bound is obtained.

Let $c_{0}$ be the constant of the best approximation of function $\varphi_{\pi / h, r}(\alpha, \beta ; t)$. For $\varepsilon>0$ we consider the set

$$
e=\left\{t \in[-h, h]:\left|\varphi_{\pi / h, r}(\alpha, \beta ; t)-c_{0}\right|>E\left(\varphi_{\pi / h, r}(\alpha, \beta ; \cdot)\right)_{L_{\infty}(\mathbb{T})}-\varepsilon\right\} .
$$

Let $f_{\mathcal{E}}(t)$ be the $2 h$-periodic function such that

$$
f_{\varepsilon}(t)= \begin{cases}(\operatorname{mes} e)^{-1} \operatorname{sign}\left(\varphi_{\pi / h, r}(\alpha, \beta ; t)-c_{0}\right), & t \in e, \\ 0, & t \in([-h, h] \backslash e),\end{cases}
$$

and $f_{\varepsilon, r}$ be the $r$ th antiderivative of $f_{\varepsilon}(t)$.

By $\tilde{f}_{\varepsilon, r}$ we denote the product $f_{\varepsilon, r}(t) \eta(t)$, where $\eta(t)$ is infinitely differentiable on $\mathbb{R}$ function such that $0 \leq \eta(t) \leq 1, t \in \mathbb{R}$ and

$$
\eta(t)= \begin{cases}1, & t \in[-\varepsilon-h, h+\varepsilon], \\ 0, & t \in(\mathbb{R} \backslash[-3 \varepsilon-h, h+3 \varepsilon]),\end{cases}
$$

(see $\left[15\right.$, p. 77]). It is clear that $\tilde{f}_{\varepsilon, r} \in L_{1}(\mathbb{R})$, supp $\tilde{f}_{\varepsilon, r}=[-3 \varepsilon-h, h+3 \varepsilon]$, and

$$
\tilde{f}_{\mathcal{E}, r}^{(r)}(t)=\sum_{j=0}^{r}\left(\begin{array}{l}
r \\
j
\end{array}\right) f_{\mathcal{\varepsilon}, r}^{(j)}(t) \eta^{(r-j)}(t) .
$$

Since for $j=0,1, \ldots, r-1$

$$
\operatorname{supp} \eta^{(r-j)}=[-h-3 \varepsilon,-h-\varepsilon] \cup[h+\varepsilon, h+3 \varepsilon],
$$

$\eta^{(r-j)}$ and $f_{\varepsilon, r}^{(j)}(t)$ are continuous on both segments $[-h-3 \varepsilon,-h-\varepsilon]$ and $[h+\varepsilon, h+3 \varepsilon]$, we can represent the function $\tilde{f}_{\varepsilon, r}^{(r)}(t)$ as $\tilde{f}_{\mathcal{\varepsilon}, r}^{(r)}(t)=f_{\varepsilon}(t) \eta(t)+\rho(t)$, where

$$
\rho(t)= \begin{cases}O(1), & t \in[-h-3 \varepsilon,-h-\varepsilon] \cup[h+\varepsilon, h+3 \varepsilon], \\ 0, & t \in(\mathbb{R} \backslash([-h-3 \varepsilon,-h-\varepsilon] \cup[h+\varepsilon, h+3 \varepsilon])),\end{cases}
$$


in particular, there exists a constant $C$ such that $|\rho(t)| \leq C$.

Now, we establish the upper estimate for the norm $\left\|\tilde{f}_{\varepsilon, r}^{(r)}\right\|_{L_{1}(\mathbb{R})}$ :

$$
\begin{aligned}
\left\|\tilde{f}_{\mathcal{\varepsilon}, r}^{(r)}\right\|_{L_{1}(\mathbb{R})} & =\int_{-h-3 \varepsilon}^{h+3 \varepsilon}\left|f_{\mathcal{\varepsilon}}(t) \eta(t)+\rho(t)\right| d t \leq \int_{-h-3 \varepsilon}^{h+3 \varepsilon}\left|f_{\varepsilon}(t) \eta(t)\right| d t+\int_{[-h-3 \varepsilon,-h-\varepsilon] \cup[h+\varepsilon, h+3 \varepsilon]}|\rho(t)| d t \\
& \leq\left\|f_{\mathcal{\varepsilon}}\right\|_{L_{1}([-h, h])}+\int_{[-h-3 \varepsilon,-h] \cup[h, h+3 \varepsilon]}\left|f_{\mathcal{\varepsilon}}(t)\right| d t+4 C \varepsilon \leq 1+C_{1} \varepsilon, \quad C_{1}>0 .
\end{aligned}
$$

Thus the function $\tilde{f}_{\varepsilon, r} /\left(1+C_{1} \varepsilon\right) \in W_{1,1}^{r}(\mathbb{R})$. Let us obtain a lower estimate for the quantity $E:=E\left(W_{1,1}^{r}(\mathbb{R}), S_{h, m}(\mathbb{R}) \cap L_{1}(\mathbb{R})\right)_{L_{1}(\mathbb{R}) ; \alpha, \beta}$.

Using the duality theorem (see, for example, [7, Theorem 1.4.9]) we can write

$$
E=\sup _{f \in W_{1,1}^{r}(\mathbb{R})} \sup _{\substack{\|g\|_{\infty ; \alpha}-1, \beta-1 \\ g \perp S_{h, m}(\mathbb{R}) \cap L_{1}(\mathbb{R})}} \int_{\mathbb{R}} f(t) g(t) d t .
$$

Based on [9, Lemmas 1.4 and 1.1] after $r$ times integration by parts we have

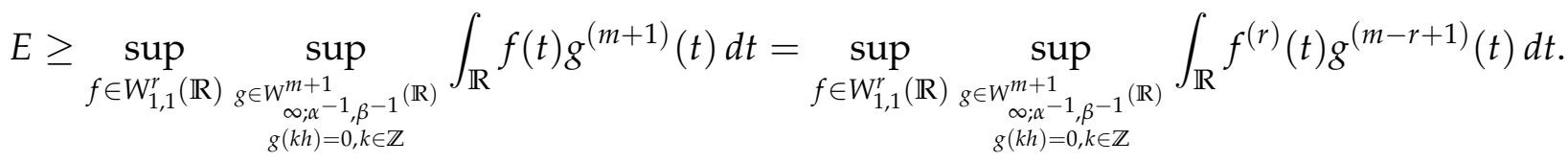

Since (with the corresponding shift) the function $\varphi_{\pi / h, m+1}(\alpha, \beta ; t) \in W_{\infty ; \alpha^{-1}, \beta^{-1}}^{m+1}(\mathbb{R})$ satisfies the conditions $\varphi_{\pi / h, m+1}(\alpha, \beta ; k h)=0, k \in \mathbb{Z}$, we obtain

$$
\begin{aligned}
E \geq \sup _{f \in W_{1,1}^{r}(\mathbb{R})} \int_{\mathbb{R}} f^{(r)}(t) \varphi_{\pi / h, r}(\alpha, \beta ; t) d t & \geq \frac{1}{1+C_{1} \varepsilon} \int_{\mathbb{R}} \tilde{f}_{\varepsilon, r}^{(r)}(t) \varphi_{\pi / h, r}(\alpha, \beta ; t) d t \\
= & \frac{1}{1+C_{1} \varepsilon}\left(\int_{-h-3 \varepsilon}^{h+3 \varepsilon}\left(f_{\varepsilon}(t) \eta(t)+\rho(t)\right) \varphi_{\pi / h, r}(\alpha, \beta ; t) d t\right) \\
= & \frac{1}{1+C_{1} \varepsilon}\left(\int_{-h}^{h} f_{\varepsilon}(t)\left(\varphi_{\pi / h, r}(\alpha, \beta ; t)-c_{0}\right) d t\right. \\
& \left.+\int_{[-h-3 \varepsilon,-h] \cup[h, h+3 \varepsilon]}\left(f_{\varepsilon} \eta(t)+\rho(t)\right) \varphi_{\pi / h, r}(\alpha, \beta ; t) d t\right) \\
\geq & \frac{1}{1+C_{1} \varepsilon}\left(\frac{1}{\operatorname{mes} e} \int_{e}\left|\varphi_{\pi / h, r}(\alpha, \beta ; t)-c_{0}\right| d t+C_{2} \varepsilon\right) \\
> & \frac{1}{1+C_{1} \varepsilon}\left(E\left(\varphi_{\pi / h, r}(\alpha, \beta ; \cdot)\right)+\left(C_{2}-1\right) \varepsilon\right) .
\end{aligned}
$$

Since $\varepsilon$ is arbitrary, we obtain the required lower bound. Thus, the theorem is proved.

\section{References}

[1] Babenko V.F. Nonsymmetric approximations in spaces of summable functions. Ukrainian Math. J. 1982, 34 (4), 331-336. doi:10.1007/BF01091584 (translation of Ukrain. Mat. Zh. 1982, 34 (4), 409-416. (in Russian))

[2] Babenko V.F., Parfinovich N.V. On the best $L_{1}$-approximations of functional classes by splines under restrictions imposed on their derivatives. Ukrainian Math. J. 1999, 51 (4), 481-491. doi:10.1007/BF02591753 (translation of Ukrain. Mat. Zh. 1999, 51 (4), 435-444. (in Russian))

[3] Babenko V.F., Parfinovich N.V. Exact values of best approximations for classes of periodic functions by splines of deficiency 2. Math. Notes. 2009, 85 (3-4), 515-527. doi:10.1134/S0001434609030237 (translation of Mat. Zametki 2009, 85 (4), 538-551. (in Russian)) 
[4] Babenko V.F., Parfinovich N.V. Nonsymmetric approximations of classes of periodic functions by splines of defect 2 and Jackson-type inequalities. Ukrainian Math. J. 2009, 61 (11), 1695-1709. doi:10.1007/s11253-010-0307-9 (translation of Ukrain. Mat. Zh. 2009, 61 (11), 1443-1454. (in Russian))

[5] Babenko V.F., Parfinovich N.V. On the exact values of the best approximations of classes of differentiable periodic functions by splines. Math. Notes. 2010, 87 (5-6), 623-635. doi:10.1134/S0001434610050032 (translation of Mat. Zametki 2010, 87 (5), 669-683. (in Russian))

[6] Doronin V.G., Ligun A.A. Upper bounds for the best one-sided approximation by splines of the classes $W^{r} L_{1}$. Math. Notes. 1976, 19 (1), 7-10. doi:10.1007/BF01147610 (translation of Mat. Zametki 1976, 19 (1), 11-17. (in Russian))

[7] Korneichuk N.P. Exact Constants in Approximation Theory. Nauka, Moscow, 1987. (in Russian)

[8] Ligun A.A. Inequalities for upper bounds of functionals. Anal. Math. 1976, 2 (1), 11-40. doi:10.1007/BF02079905

[9] Magaril-Il'yaev G.G. On best approximation by splines of function classes on the line. Proc. Steklov Inst. Math. 1993, 194, 153-164. (translation of Tr. Mat. Inst. Steklova 1992, 194, 148-159. (in Russian))

[10] Magaril-Il'yaev G.G., Tikhomirov V.M. On approximation of functional classes on the real line by splines and entire functions. In: Function Spaces and Their Application to Differential Equations. Collection of Scientific Papers. RUDN, Moskow, 1992, 116-129. (in Russian)

[11] Parfinovych N.V. Exact values of the best $(\alpha, \beta)$-approximations for the classes of convolutions with kernels that do not increase the number of sign changes. Ukrainian Math. J. 2018, 69 (8), 1248-1261. doi:10.1007/s11253-017-1428-1 (translation of Ukrain. Mat. Zh. 2017, 69 (8), 1073-1083. (in Russian))

[12] Parfinovych N.V. On extremal subspaces for the widths of classes of convolutions. Res. Math. 2017,22 (8), 68-79. doi: $10.15421 / 241708$

[13] Stain E.M. Functions of exponential type. Ann. Math. 1957, 65 (3), 582-592. doi:10.2307/1970066

[14] Tikhomirov V.M. On the n-dimensional widthes of some functional classes. Dokl. Akad. Nauk USSR. 1960, 130 (4), 734-737. (in Russian)

[15] Vladimirov V.S. Equations of Mathematical Physics. Nauka, Moscow, 1967. (in Russian)

Received 02.03.2021

Revised 03.11.2021

Парфінович Н.В. Несиметричні наближення функиіональних класів сплайнами на дійсній осі // Карпатські матем. публ. - 2021. - Т.13, №3. - С. 831-837.

Нехай $S_{h, m}, h>0, m \in \mathbb{N}$ - простори поліноміальних сплайнів порядку $m$ дефекту 13 вузлами в точках $k h, k \in \mathbb{Z}$.

Отримано точні значення найкраших $(\alpha, \beta)$-наближень просторами $S_{h, m} \cap L_{1}(\mathbb{R})$ у просторі $L_{1}(\mathbb{R})$ для класів $W_{1,1}^{r}(\mathbb{R}), r \in \mathbb{N}$, функцій, визначених на всій дійсній прямій, інтегрованих на $\mathbb{R}$ і таких, що $r$-ті похідні належать одиничній кулі $L_{1}(\mathbb{R})$.

Ці результати узагальнюють відомі результати Г.Г. Магарила-Ілляєва та В.М. Тихомирова шодо точних значень найкраших наближень класів $W_{1,1}^{r}(\mathbb{R})$ сплайнами з $S_{h, m} \cap L_{1}(\mathbb{R})$ (випадок $\alpha=\beta=1)$, а також $є$ неперіодичними аналогами В.Ф. Бабенка щодо найкращих несиметричних наближень класів $W_{1}^{r}(\mathbb{T}) 2 \pi$-періодичних функцій з $r$-тою похідною, що належить до одиничної кулі простору $L_{1}(\mathbb{T})$ періодичними поліноміальніми сплайнами мінімального дефекту.

Як наслідок основного результату, ми отримуємо точні значення найкращих односторонніх наближень класів $W_{1}^{r}$ поліноміальними сплайнами з $S_{h, m}(\mathbb{T})$. Цей результат є періодичним аналогом результатів А.А. Аігуна і В.Г. Аороніна про найкращі односторонні наближення класів $W_{1}^{r}$ просторами $S_{h, m}(\mathbb{T})$.

Ключові слова і фрази: найкраще $L_{1}$-наближення, одностороннє наближення, несиметричне наближення, поліноміальний сплайн, функціональний клас. 